\title{
Quantifying Weak Hydrogen Bonding in Uracil and 4-cyano-4'-ethynylbiphenyl: A Combined Computational and Experimental Investigation of NMR Chemical Shifts in the Solid State
}

\author{
Anne-Christine Uldry, John M. Griffin, Jonathan R. Yates, \\ Marta Pérez-Torralba, M. Dolores Santa María, Amy L. Webber, \\ Maximus L. L. Beaumont, Ago Samoson, Rosa María Claramunt, \\ Chris J. Pickard and Steven P. Brown*
}

The following Tables list the calculated absolute chemical shieldings and NICS values for all atoms. Full synthetic details to obtain $\mathbf{2}$ and ${ }^{15} \mathrm{~N}_{-}{ }^{13} \mathrm{C}_{2}-\mathbf{2}$ are also given.

\section{Further computational results}

The employed labelling for 4-cyano-4'-ethynylbiphenyl is given in Figure S1. The calculation details are given in the Computational details section of the main text. The GIPAW formalism enables the use of pseudopotentials in the determination of NMR properties, thereby reducing the size of the basis set otherwise required and thus allowing calculations of larger systems. The all-electron properties are reconstructed at the nucleus, which ensures that the shieldings calculated with pseudopotentials have the all-electron accuracy. Though an earlier implementation within the PARATEC code $^{1}$ used the norm-conserving Trouiller-Martins pseudopotentials, the formalism has been extended in CASTEP to allow the use of ultra-soft pseudopotentials (Ref. 81 to 83 of the main text).

The shieldings for 1 were obtained in under 40 hours on the $1.6 \mathrm{GHz}$ Itanium2 at Warwick University; the calculations were parallelized over 4 CPU slots. The shieldings for 2 were obtained in just over 127 hours on the AMD Opteron cluster at St Andrews University; in this case the calculations were parallelized over 16 nodes.

Tables S1 to S4 list the calculated shieldings and NICSs. Experimental and computed chemical shifts $\delta_{\text {iso }}$ are given in the main text, as usual, with respect to a reference (e.g., TMS for ${ }^{1} \mathrm{H}$ and ${ }^{13} \mathrm{C}$ ), i.e., $\delta_{\text {iso }}=\sigma_{\text {ref }}-\sigma_{\text {iso }}$, where $\sigma_{\text {ref }}$ is the shielding in the reference and $\sigma_{\text {iso }}$ the absolute shielding in the considered sample. In principle, shielding values could be calculated for both the reference and the considered sample in order to compute the chemical shift. In practice, the established procedure (Refs. 51, 53, 87 and 88 of the main text) is that only $\sigma_{\text {iso }}$ is calculated. The reference $\sigma_{\text {ref }}$ is obtained by fitting a straight line of slope -1 to the experimental shifts versus calculated shieldings plot, and are given in 
Table S1. Uracil. Calculated isotropic chemical shielding $\sigma_{i s o} / \mathrm{ppm}$

\begin{tabular}{|cccccc|}
\hline Site & Cryst. & Plane & Mol. & Rel. Mol. $^{a}$ & -NICS \\
\hline H1 & 18.0 & 17.2 & 22.3 & 23.0 & -0.1 \\
H2 & 18.5 & 17.8 & 23.2 & 23.9 & -0.01 \\
H3 & 22.5 & 21.6 & 23.6 & 23.6 & -0.2 \\
H4 & 24.2 & 23.4 & 25.6 & 25.7 & -0.3 \\
\hline C1 & -1.9 & -2.3 & 3.0 & 8.0 & -0.6 \\
C2 & 17.9 & 17.3 & 19.8 & 21.0 & -0.4 \\
C3 & 18.7 & 19.7 & 29.6 & 30.4 & -0.3 \\
C4 & 67.7 & 68.8 & 67.3 & 66.3 & -0.3 \\
\hline N1 & 54.9 & 55.3 & 54.6 & 57.3 & -0.5 \\
N2 & 70.9 & 72.5 & 89.9 & 91.4 & -0.3 \\
\hline O1 & -12.9 & -11.7 & -146.3 & -117.5 & -1.0 \\
O2 & 3.9 & 5.5 & -24.5 & -13.3 & -0.6 \\
\hline
\end{tabular}

${ }^{a}$ All atoms relaxed

the main text.

The absolute chemical shielding for the proton-only and all-atom-relaxed isolated molecules of 4-cyano-4'-ethynylbiphenyl are listed in Table S4. When all the atoms are relaxed, the four molecules tend to adopt the same shape with similar relative orientations of the two benzene rings. The torsion angle $\phi$ is defined here as the dihedral angle formed by the three bonds $\mathrm{C} 4-\mathrm{C} 5, \mathrm{C} 5-\mathrm{C} 8$ and $\mathrm{C} 8-\mathrm{C} 9$. In the (optimized) crystal, $\phi$ is $47.5^{\circ}, 41.9^{\circ}, 40.4^{\circ}$ and $17.0^{\circ}$ for molecule 1 to 4 , respectively. When left to relax, $\phi$ becomes $36.2^{\circ}, 41.8^{\circ}$, $38.8^{\circ}$ and $30.0^{\circ}$, respectively.

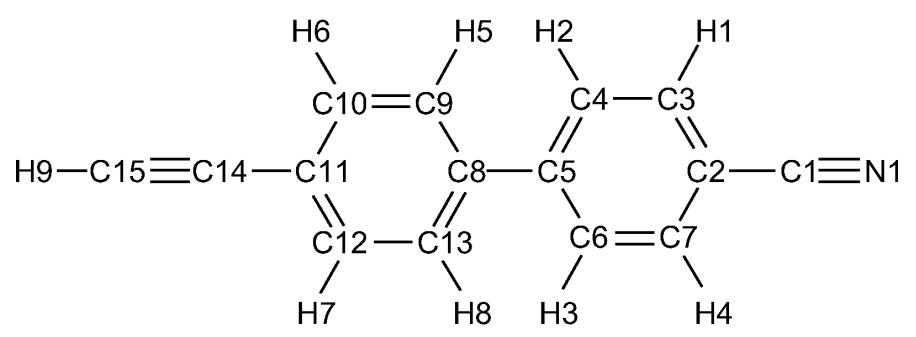

Figure S1. 4-cyano-4'-ethynylbiphenyl 
Table S2. 4-cyano-4'-ethynylbiphenyl. Calculated isotropic chemical shielding $\sigma_{i s o} / \mathrm{ppm}$ in the crystal

\begin{tabular}{|ccccc|}
\hline Site & \multicolumn{4}{c|}{ Crystal } \\
\hline & Mol 1 & Mol 2 & Mol 3 & Mol4 \\
H4 & 22.8 & 25.4 & 23.3 & 24.3 \\
H6 & 23.0 & 23.2 & 23.9 & 25.9 \\
H3 & 23.4 & 23.7 & 25.4 & 24.7 \\
H2 & 23.5 & 23.6 & 23.0 & 23.6 \\
H1 & 23.8 & 25.1 & 22.9 & 22.5 \\
H7 & 24.4 & 24.0 & 25.4 & 25.6 \\
H8 & 24.8 & 24.8 & 24.4 & 23.4 \\
H5 & 25.0 & 23.0 & 23.8 & 23.4 \\
H9 & 25.1 & 25.7 & 24.8 & 24.9 \\
\hline C5 & 19.5 & 20.7 & 21.3 & 27.0 \\
C8 & 24.6 & 27.7 & 27.7 & 32.3 \\
C3 & 30.9 & 33.0 & 31.3 & 31.4 \\
C7 & 32.4 & 32.7 & 31.0 & 33.4 \\
C12 & 32.5 & 31.9 & 33.5 & 32.2 \\
C10 & 33.4 & 33.1 & 31.5 & 31.1 \\
C1 & 37.1 & 37.4 & 36.5 & 38.8 \\
C4 & 37.4 & 37.8 & 37.3 & 40.6 \\
C6 & 37.5 & 36.6 & 39.0 & 42.1 \\
C13 & 38.3 & 39.5 & 38.4 & 42.2 \\
C9 & 39.5 & 38.3 & 37.8 & 42.2 \\
C11 & 47.7 & 46.1 & 46.5 & 44.9 \\
C2 & 61.0 & 61.4 & 60.5 & 61.2 \\
C15 & 76.0 & 77.8 & 76.4 & 75.8 \\
C14 & 82.1 & 81.8 & 80.5 & 82.3 \\
\hline N1 & -51.3 & -52.6 & -54.2 & -54.5 \\
\hline & & & &
\end{tabular}


Table S3. 4-cyano-4'-ethynylbiphenyl. Calculated isotropic chemical shielding $\sigma_{i s o} / \mathrm{ppm}$ for the isolated chains and molecules, and corresponding NICS

\begin{tabular}{|c|cccc|cccc|ccccc|}
\hline Site & \multicolumn{7}{|c|}{ Chain } & \multicolumn{7}{|c|}{ Mol. } & \multicolumn{4}{c|}{-NICS } \\
\hline & 1 & 2 & 3 & 4 & 1 & 2 & 3 & 4 & 1 & 2 & 3 & 4 \\
H4 & 22.8 & 22.8 & 22.8 & 22.9 & 22.8 & 22.8 & 22.8 & 22.9 & 0.0 & 0.0 & 0.0 & 0.1 \\
H6 & 23.3 & 23.3 & 23.3 & 23.3 & 23.2 & 23.2 & 23.2 & 23.3 & 0.1 & 0.1 & 0.1 & 0.0 \\
H3 & 23.4 & 23.3 & 23.4 & 22.8 & 23.5 & 23.4 & 23.4 & 22.8 & 0.0 & 0.0 & 0.0 & 0.0 \\
H2 & 23.4 & 23.4 & 23.3 & 22.8 & 23.4 & 23.4 & 23.3 & 22.8 & 0.0 & 0.0 & 0.0 & 0.0 \\
H1 & 22.8 & 22.8 & 22.8 & 22.9 & 22.8 & 22.8 & 22.8 & 22.9 & 0.0 & 0.0 & 0.0 & -0.1 \\
H7 & 23.3 & 23.2 & 23.3 & 23.3 & 23.2 & 23.2 & 23.2 & 23.3 & 0.1 & 0.1 & 0.1 & 0.0 \\
H8 & 23.7 & 23.6 & 23.6 & 23.0 & 23.6 & 23.5 & 23.5 & 23.0 & 0.0 & 0.0 & 0.0 & 0.0 \\
H5 & 23.7 & 23.6 & 23.6 & 23.0 & 23.6 & 23.5 & 23.5 & 22.9 & 0.0 & 0.0 & 0.0 & 0.0 \\
H9 & 25.2 & 25.3 & 25.1 & 24.9 & 26.4 & 26.4 & 26.3 & 26.3 & 0.9 & 0.8 & 0.9 & 1.0 \\
\hline C5 & 18.1 & 19.1 & 19.6 & 25.0 & 20.0 & 20.9 & 21.4 & 26.9 & 0.1 & 0.1 & 0.1 & 0.0 \\
C8 & 27.2 & 28.2 & 28.4 & 34.1 & 25.0 & 26.1 & 26.3 & 31.9 & 0.2 & 0.2 & 0.2 & -0.1 \\
C3 & 33.5 & 33.6 & 33.7 & 33.8 & 33.5 & 33.6 & 33.6 & 33.8 & 0.0 & 0.0 & 0.0 & 0.0 \\
C7 & 33.9 & 33.8 & 33.1 & 33.4 & 33.9 & 33.8 & 33.1 & 33.4 & 0.0 & 0.0 & 0.0 & 0.0 \\
C12 & 32.8 & 33.0 & 34.7 & 33.0 & 32.6 & 32.8 & 34.5 & 32.8 & 0.0 & 0.0 & 0.0 & 0.0 \\
C10 & 34.9 & 33.9 & 32.8 & 34.1 & 34.6 & 33.7 & 32.7 & 33.9 & 0.0 & 0.0 & 0.0 & 0.1 \\
C1 & 40.9 & 40.5 & 40.7 & 41.2 & 39.8 & 39.9 & 39.8 & 40.0 & -0.1 & -0.1 & -0.1 & 0.2 \\
C4 & 39.4 & 40.3 & 40.2 & 43.8 & 39.6 & 40.4 & 40.3 & 43.7 & 0.1 & 0.1 & 0.1 & 0.0 \\
C6 & 39.7 & 39.5 & 40.1 & 43.2 & 39.9 & 39.6 & 40.2 & 43.1 & 0.1 & 0.1 & 0.1 & 0.0 \\
C13 & 41.0 & 41.4 & 41.3 & 43.2 & 41.6 & 41.1 & 41.0 & 43.1 & 0.0 & 0.0 & 0.0 & 0.0 \\
C9 & 41.0 & 41.1 & 41.4 & 44.5 & 41.7 & 40.8 & 41.1 & 44.4 & 0.3 & 0.3 & 0.3 & 0.3 \\
C11 & 42.5 & 42.0 & 42.3 & 41.0 & 45.4 & 44.8 & 45.3 & 44.4 & 0.0 & 0.0 & 0.0 & 0.1 \\
C2 & 58.4 & 58.5 & 58.3 & 59.2 & 55.9 & 56.0 & 55.6 & 56.2 & 0.0 & 0.0 & 0.0 & 0.0 \\
C15 & 77.5 & 77.4 & 76.9 & 75.0 & 87.7 & 87.2 & 87.3 & 86.5 & 0.0 & 0.0 & 0.0 & 0.1 \\
C14 & 87.4 & 87.3 & 87.2 & 86.8 & 85.3 & 85.3 & 85.2 & 85.0 & 0.0 & 0.0 & 0.0 & 0.0 \\
\hline N1 & -59.3 & -59.4 & -58.7 & -57.7 & -71.5 & -71.1 & -71.2 & -71.1 & -0.2 & -0.2 & -0.2 & -0.2 \\
\hline
\end{tabular}


Table S4. 4-cyano-4'-ethynylbiphenyl. Calculated isotropic chemical shielding $\sigma_{i s o} / \mathrm{ppm}$ for the proton-relaxed and fully-relaxed isolated molecules

\begin{tabular}{|c|cccc|cccc|}
\hline Site & \multicolumn{4}{|c|}{ H-Rel. Mol. } & \multicolumn{4}{c|}{ All Rel. Mol. } \\
\hline & 1 & 2 & 3 & 4 & 1 & 2 & 3 & 4 \\
H4 & 22.8 & 22.8 & 22.8 & 22.9 & 22.8 & 22.8 & 22.8 & 22.8 \\
H6 & 23.2 & 23.2 & 23.2 & 23.2 & 23.2 & 23.2 & 23.2 & 23.2 \\
H3 & 23.4 & 23.3 & 23.3 & 22.8 & 23.2 & 23.3 & 23.3 & 23.1 \\
H2 & 23.4 & 23.4 & 23.3 & 22.8 & 23.2 & 23.4 & 23.3 & 23.1 \\
H1 & 22.8 & 22.8 & 22.8 & 22.9 & 22.8 & 22.8 & 22.8 & 22.8 \\
H7 & 23.2 & 23.2 & 23.2 & 23.2 & 23.2 & 23.2 & 23.2 & 23.2 \\
H8 & 23.6 & 23.5 & 23.5 & 23.0 & 23.4 & 23.5 & 23.5 & 23.2 \\
H5 & 23.6 & 23.5 & 23.4 & 22.9 & 23.4 & 23.5 & 23.4 & 23.3 \\
H9 & 26.6 & 26.5 & 26.5 & 26.6 & 26.5 & 26.6 & 26.5 & 26.5 \\
\hline C5 & 19.6 & 20.6 & 21.0 & 26.8 & 21.9 & 20.9 & 21.0 & 23.4 \\
C8 & 24.8 & 25.6 & 26.0 & 31.9 & 27.0 & 26.0 & 26.0 & 28.5 \\
C3 & 33.7 & 33.7 & 33.9 & 33.8 & 33.5 & 33.6 & 33.9 & 33.4 \\
C7 & 34.1 & 33.7 & 33.2 & 33.4 & 33.3 & 33.5 & 33.2 & 33.3 \\
C12 & 32.6 & 32.9 & 34.3 & 32.7 & 32.8 & 32.8 & 34.3 & 33.2 \\
C10 & 34.6 & 33.9 & 32.6 & 33.8 & 33.2 & 33.5 & 32.6 & 33.0 \\
C1 & 39.8 & 39.8 & 39.7 & 39.9 & 40.0 & 40.0 & 39.7 & 40.0 \\
C4 & 39.8 & 40.5 & 40.3 & 43.8 & 40.5 & 40.2 & 40.3 & 41.3 \\
C6 & 39.9 & 39.8 & 40.1 & 43.5 & 40.6 & 40.0 & 40.1 & 40.4 \\
C13 & 40.7 & 40.9 & 40.9 & 43.1 & 41.2 & 40.9 & 40.9 & 42.1 \\
C9 & 40.9 & 40.7 & 41.2 & 44.5 & 41.0 & 40.6 & 41.2 & 41.8 \\
C11 & 45.0 & 44.9 & 45.0 & 44.3 & 44.4 & 44.6 & 45.0 & 44.3 \\
C2 & 55.4 & 55.5 & 55.4 & 55.4 & 55.0 & 55.1 & 55.4 & 55.0 \\
C15 & 87.9 & 87.4 & 87.6 & 86.7 & 87.4 & 87.7 & 87.6 & 87.2 \\
C14 & 85.2 & 85.2 & 85.1 & 84.9 & 85.3 & 85.3 & 85.1 & 85.2 \\
\hline N1 & -71.4 & -71.0 & -71.1 & -71.1 & -71.3 & -71.2 & -71.1 & -71.5 \\
\hline
\end{tabular}




\section{Synthesis of 2: Further details}

General: Melting points were determined by DSC on a Seiko DSC 220C connected to a Model SSC5200H Disk Station. The heating rates were $5^{\circ} \mathrm{min}^{-1}$ with $\mathrm{N}_{2}$ as the purge gas. Unless otherwise stated, column chromatography was performed on silica gel (Merck 60, 70-230 mesh). In addition, Mass spectra (HRMS) at $70 \mathrm{eV}$ using electron impact mode was performed with a VG AUTOSPEC spectrometer by Laboratorio de Espectrometría de Masas-UAM, Madrid.

Solution NMR parameters: NMR spectra were recorded on a Bruker DRX 400 (9.4 Tesla, $400.13 \mathrm{MHz}$ for ${ }^{1} \mathrm{H}$ and $100.62 \mathrm{MHz}$ for ${ }^{13} \mathrm{C}$ ) spectrometer with a 5 -mm inverse-detection $\mathrm{H}$-X probe equipped with a z-gradient coil, at $300 \mathrm{~K}$. Chemical shifts ( $\delta$ in ppm) are given from internal solvent, $\mathrm{CDCl}_{3} 7.26$ for ${ }^{1} \mathrm{H}$ and 77.0 for ${ }^{13} \mathrm{C}$. Typical parameters for ${ }^{1} \mathrm{H}$ NMR spectra were spectral width $4800 \mathrm{~Hz}$, pulse width $7.5 \mu$ s at an attenuation level of $0 \mathrm{~dB}$ and resolution $0.29 \mathrm{~Hz}$ per point. Typical parameters for ${ }^{13} \mathrm{C}$ NMR spectra were spectral width $20600 \mathrm{~Hz}$, pulse width $10.6 \mu \mathrm{s}$ at an attenuation level of $-6 \mathrm{~dB}$ and resolution $0.63 \mathrm{~Hz}$ per point; WALTZ-16 was used for broadband proton decoupling; the FIDS were multiplied by an exponential weighting ( $\mathrm{lb}=1 \mathrm{~Hz}$ ) before Fourier transformation. 2D inverse proton detected heteronuclear shift correlation spectra, $\left({ }^{1} \mathrm{H}-{ }^{13} \mathrm{C}\right)$ gs-HMQC and $\left({ }^{1} \mathrm{H}_{-}{ }^{13} \mathrm{C}\right)$ gs-HMBC were acquired and processed using standard Bruker NMR software in non-phase-sensitive mode and were carried out to assign the ${ }^{1} \mathrm{H}$ and ${ }^{13} \mathrm{C}$ signals. Gradient selection was achieved through a $5 \%$ sine truncated shaped pulse gradient of $1 \mathrm{~ms}$. Selected parameters for $\left({ }^{1} \mathrm{H}_{-}{ }^{13} \mathrm{C}\right)$ gs-HMQC spectra were spectral width $3900 \mathrm{~Hz}$ for ${ }^{1} \mathrm{H}$ and 17.48 $\mathrm{kHz}$ for ${ }^{13} \mathrm{C}, 512 \times 128$ data set, 2 co-added transients and relaxation delay $1 \mathrm{~s}$. Parameters for $\left({ }^{1} \mathrm{H}_{-}{ }^{13} \mathrm{C}\right)$ gs-HMBC spectra were spectral width $3200-3700 \mathrm{~Hz}$ for ${ }^{1} \mathrm{H}$ and $22.14 \mathrm{kHz}$ for ${ }^{13} \mathrm{C}, 1024 \times 256$ data set, 4 co-added transients, relaxation delay $1 \mathrm{~s}$ and $60 \mathrm{~ms}$ delay for the evolution of the ${ }^{13} \mathrm{C}^{-1} \mathrm{H}$ long-range coupling.

4-Cyano-4'-iodobiphenyl (3).

IUPAC name: 4'-iodobiphenyl-4-carbonitrile (3).

Prepared using the procedure described in Ref. 2. 4,4'-Diiodobiphenyl (2.0 g, $4.93 \mathrm{mmol})$, cuprous cyanide $(0.44 \mathrm{~g}, 4.93 \mathrm{mmol})$, and dimethylformamide $(60 \mathrm{~mL})$ were refluxed for $3.5 \mathrm{~h}$. After cooling, $140 \mathrm{~mL}$ of ferric chloride solution $\left(0.20 \mathrm{~g}\right.$ of hydrated $\mathrm{FeCl}_{3}$ and 50 $\mathrm{mL}$ of concentrated $\mathrm{HCl}$ in $300 \mathrm{~mL}$ of water) was added to the reaction. The resulting mixture was heated at $60-70{ }^{\circ} \mathrm{C}$ for $30 \mathrm{~min}$. After cooling, the mixture was extracted with toluene $(80 \mathrm{~mL} \times 3)$. The reddish toluene layer was then extracted with $10 \% \mathrm{HCl}(200$ $\mathrm{mL})$, water $(80 \mathrm{~mL})$, and $5 \% \mathrm{NaOH}(200 \mathrm{~mL})$. The toluene layer was dried over $\mathrm{MgSO}_{4}$ and filtered; after evaporation of the solvent, the resulting solid was chromatographed with the following eluents: hexane (4,4'-diiodobiphenyl), 1:20 AcOEt-hexane (3) and 1:5 AcOEthexane (4,4'-dicyanobiphenyl). The yield of $\mathbf{3}$ was $49 \%$ (732 mg). M.p. $179.6{ }^{\circ} \mathrm{C} .{ }^{1} \mathrm{H}$ NMR $\left(\mathrm{CDCl}_{3}\right) \delta 7.82(\mathrm{~m}, \mathrm{H} 6 / \mathrm{H} 7), 7.73(\mathrm{~m}, \mathrm{H} 1 / \mathrm{H} 4), 7.65(\mathrm{~m}, \mathrm{H} 2 / \mathrm{H} 3), 7.32(\mathrm{~m}, \mathrm{H} 5 / \mathrm{H} 8) .{ }^{13} \mathrm{C}$ NMR $\left(\mathrm{CDCl}_{3}\right) \delta 144.5(\mathrm{C} 5), 138.6(\mathrm{C} 8), 138.2$ (C10/C12), 132.7 (C3/C7), 128.9 (C9/C13), 127.5 (C4/C6), 118.7 (C1), 111.4 (C2), 94.8 (C11). 
4-Cyano-4'-trimethylsilylethynylbiphenyl (4).

IUPAC name: 4'-[(trimethylsilyl)ethynyl]biphenyl-4-carbonitrile (4).

Prepared using the procedure described in Ref. 3. To a mixture of $\mathbf{3}(0.53 \mathrm{~g}, 1.72$ $\mathrm{mmol})$ and trimethylsilylacetylene $(0.21 \mathrm{~g}, 2.06 \mathrm{mmol})$ in triethylamine $(10 \mathrm{~mL})$ were added bis(triphenylphosphine)palladium (II) dichloride (24.2 $\mathrm{mg}, 0.034 \mathrm{mmol})$ and copper(I) iodide (3.3 $\mathrm{mg}, 0.017 \mathrm{mmol}$ ). The reaction mixture was stirred at room temperature, under argon atmosphere for $41 \mathrm{~h}$. The triethylamine was evaporated and the crude was washed with ethyl acetate. The solvent was evaporated, and the crude product was purified by column chromatography using 1:50 AcOEt-hexane as eluent, to give 4 (415 mg, 88\%). M.p. $114.7{ }^{\circ} \mathrm{C} .{ }^{1} \mathrm{H} \mathrm{NMR}\left(\mathrm{CDCl}_{3}\right) \delta 7.73(\mathrm{~m}, \mathrm{H} 1 / \mathrm{H} 4), 7.68$ (m, H2/H3), 7.57 (m, H6/H7), 7.53 (m, H5/H8), $0.27\left(\mathrm{~s}, \mathrm{CH}_{3}\right) .{ }^{13} \mathrm{C} \mathrm{NMR}\left(\mathrm{CDCl}_{3}\right) \delta 144.7$ (C5), $138.9(\mathrm{C} 8), 132.6(\mathrm{C} 3 / \mathrm{C} 7$, C10/C12), 127.6 (C4/C6), 127.0 (C9/C13), 123.6 (C11), 118.8 (C1), 111.3 (C2), 104.3 (C14), 96.1 (C15), -0.09 $\left(\mathrm{CH}_{3}\right)$. HR-EI-MS $\mathrm{m} / z$ calcd for $\mathrm{C}_{18} \mathrm{H}_{17} \mathrm{NSi} 275.1130$, found 275.1142 .

4-Cyano-4'-ethynylbiphenyl (2).

IUPAC name: 4'-ethynylbiphenyl-4-carbonitrile (2).

Prepared using the procedure described in Ref. 3. The solution of $4(0.33 \mathrm{~g}, 1.19 \mathrm{mmol})$ in methanol $(20 \mathrm{~mL}), 1 \mathrm{~N}$ aqueous potassium hydroxide solution $(1.2 \mathrm{~mL})$ was added and the mixture was stirred at room temperature for $1 \mathrm{~h}$. After removal of methanol, the product was extracted with dichloromethane and recrystallized from dichloromethane-hexane to give $2(238 \mathrm{mg}, 99 \%)$. M.p. $205.6{ }^{\circ} \mathrm{C} .{ }^{1} \mathrm{H} \mathrm{NMR}\left(\mathrm{CDCl}_{3}\right) \delta 7.74(\mathrm{~m}, \mathrm{H} 1 / \mathrm{H} 4), 7.68$ (m,

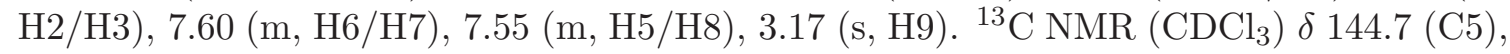
139.4 (C8), 132.8 (C10/C12), 132.7 (C3/C7), 127.6 (C4/C6), 127.1 (C9/C13), 122.6 (C11), 118.7 (C1), 111.4 (C2), 83.0 (C14), 78.7 (C15). HR-EI-MS m/z calcd for $\mathrm{C}_{15} \mathrm{H}_{9} \mathrm{~N} 203.0735$, found 203.0725 .

4- $\left({ }^{15} \mathrm{~N}\right)$ Cyano-4'-iodobiphenyl $\left({ }^{15} \mathrm{~N}-3\right)$. IUPAC name: 4'-iodobiphenyl-4- $\left({ }^{15} \mathrm{~N}\right)$ carbonitrile $\left({ }^{15} \mathrm{~N}-3\right)$.

Prepared using the procedure described in Ref. 2. 4,4'-Diiodobiphenyl (2.0 g, $4.93 \mathrm{mmol})$, $\left({ }^{15} \mathrm{~N}\right)$-cuprous cyanide $\left({ }^{15} \mathrm{~N} 98 \%, 0.45 \mathrm{~g}, 4.93 \mathrm{mmol}\right)$, and dimethylformamide $(60 \mathrm{~mL})$ were refluxed for $3.5 \mathrm{~h}$. After cooling, $140 \mathrm{~mL}$ of ferric chloride solution $(200 \mathrm{~g}$ of hydrated $\mathrm{FeCl}_{3}$ and $50 \mathrm{~mL}$ of concentrated $\mathrm{HCl}$ in $300 \mathrm{~mL}$ of water) was added to the reaction. The resulting mixture was heated at $60-70{ }^{\circ} \mathrm{C}$ for $30 \mathrm{~min}$. After cooling, the mixture was extracted with toluene $(80 \mathrm{~mL} \times 3)$. The reddish toluene layer was then extracted with $10 \% \mathrm{HCl}(200 \mathrm{~mL})$, water $(80 \mathrm{~mL})$, and $5 \% \mathrm{NaOH}(200 \mathrm{~mL})$. The toluene layer was dried over $\mathrm{MgSO}_{4}$ and filtered; after evaporation of the solvent, the resulting solid was chromatographed with the following eluents: hexane (4,4'-diiodobiphenyl), 1:20 AcOEt-hexane $\left({ }^{\mathbf{1 5}} \mathrm{N}-3\right)$ and 1:5 AcOEt-hexane $\left(4,4{ }^{\prime}-\left({ }^{15} \mathrm{~N}_{2}\right)\right.$ dicyanobiphenyl). The yield of ${ }^{\mathbf{1 5}} \mathrm{N}-\mathbf{3}$ was $31 \%$ (463 mg). ${ }^{1} \mathrm{H}$ NMR $\left(\mathrm{CDCl}_{3}\right) \delta 7.82$ (m, H6/H7), 7.73 (m, H1/H4), 7.65 (m, H2/H3 ), 7.32 (m, H5/H8). ${ }^{13} \mathrm{C} \mathrm{NMR}\left(\mathrm{CDCl}_{3}\right) \delta 144.5$ (C5), 138.6 (C8), 138.2 (C10/C12), 132.7 (C3/C7), $128.9(\mathrm{C} 9 / \mathrm{C} 13), 127.5(\mathrm{C} 4 / \mathrm{C} 6), 118.7\left(\mathrm{~d},{ }^{1} \mathrm{~J}_{\mathrm{N} 1}=17.7 \mathrm{~Hz}, \mathrm{C} 1\right), 111.4\left(\mathrm{~d},{ }^{2} \mathrm{~J}_{\mathrm{N} 1}=2.8 \mathrm{~Hz}\right.$, C2), 94.8 (C11). 
4- $\left({ }^{15} \mathrm{~N}\right)$ Cyano-4'-[(trimethylsilyl) $\left({ }^{13} \mathrm{C}_{2}\right)$ ethynyl]biphenyl $\left({ }^{15} \mathrm{~N}-{ }^{13} \mathrm{C}_{2}-4\right)$. IUPAC name: 4'-[(trimethylsilyl $)\left({ }^{13} \mathrm{C}_{2}\right)$ ethynyl] biphenyl-4- $\left({ }^{15} \mathrm{~N}\right)$ carbonitrile $\left({ }^{15} \mathrm{~N}_{-}{ }^{13} \mathrm{C}_{2}-4\right)$.

Prepared using the procedure described in Ref. 3. To a mixture of ${ }^{\mathbf{1 5}} \mathrm{N}-\mathbf{3}(0.50 \mathrm{~g}, 1.63$ mmol $)$ and trimethylsilyl $\left({ }^{13} \mathrm{C}_{2}\right)$ acetylene $\left({ }^{13} \mathrm{C}_{2} 99 \%, 0.20 \mathrm{~g}, 1.96 \mathrm{mmol}\right)$ in triethylamine (9.5 mL) were added bis(triphenylphosphine)palladium (II) dichloride (23 $\mathrm{mg}, 0.033 \mathrm{mmol}$ ) and copper(I) iodide $(3.1 \mathrm{mg}, 0.016 \mathrm{mmol})$. The reaction mixture was stirred at room temperature, under argon atmosphere for $42 \mathrm{~h}$. The triethylamine was evaporated and the crude was washed with ethyl acetate. The solvent was evaporated, and the crude product was purified by column chromatography using 1:50 AcOEt-hexane as eluent, to afford ${ }^{15} \mathrm{~N}$ ${ }^{13} \mathbf{C}_{\mathbf{2}} \mathbf{- 4}(394 \mathrm{mg}, 87 \%) .{ }^{1} \mathrm{H} \mathrm{NMR}\left(\mathrm{CDCl}_{3}\right) \delta 7.73(\mathrm{~m}, \mathrm{H} 1 / \mathrm{H} 4), 7.67$ (m, H2/H3), 7.59-7.52 $(\mathrm{m}, \mathrm{H} 6 / \mathrm{H} 7, \mathrm{H} 5 / \mathrm{H} 8), 0.27\left(\mathrm{~d},{ }^{3} \mathrm{~J}_{\mathrm{C} 15}=2.7 \mathrm{~Hz}, \mathrm{CH}_{3}\right) .{ }^{13} \mathrm{C} \mathrm{NMR}\left(\mathrm{CDCl}_{3}\right) \delta 144.7(\mathrm{C} 5)$, 138.9 (C8), 132.6 (C3/C7, C10/C12), 127.6 (C4/C6), 127.0 (d, $\left.{ }^{3} \mathrm{~J}_{\mathrm{C} 14}=5.3 \mathrm{~Hz}, \mathrm{C} 9 / \mathrm{C} 13\right)$, $123.6\left(\mathrm{dd},{ }^{1} \mathrm{~J}_{\mathrm{C} 14}=84.6 \mathrm{~Hz},{ }^{2} \mathrm{~J}_{\mathrm{C} 15}=9.6 \mathrm{~Hz}, \mathrm{C} 11\right), 118.8\left(\mathrm{~d},{ }^{1} \mathrm{~J}_{\mathrm{N} 1}=18.0 \mathrm{~Hz}, \mathrm{C} 1\right), 111.3$ $\left(\mathrm{d},{ }^{2} \mathrm{~J}_{\mathrm{N} 1}=3.2 \mathrm{~Hz}, \mathrm{C} 2\right), 104.4\left(\mathrm{~d},{ }^{1} \mathrm{~J}_{\mathrm{C} 15}=135.5 \mathrm{~Hz}, \mathrm{C} 14\right), 96.1\left(\mathrm{~d},{ }^{1} \mathrm{~J}_{\mathrm{C} 14}=135.5 \mathrm{~Hz}, \mathrm{C} 15\right)$, $-0.09\left(\mathrm{~d},{ }^{2} \mathrm{~J}_{\mathrm{C} 15}=5.3 \mathrm{~Hz}, \mathrm{CH}_{3}\right)$.

4- $\left({ }^{15} \mathrm{~N}\right)$ Cyano-4'-( $\left.{ }^{13} \mathrm{C}_{2}\right)$ ethynylbiphenyl $\left({ }^{15} \mathrm{~N}^{13} \mathrm{C}_{2}-2\right)$.

IUPAC name: $4^{\prime}-\left({ }^{13} \mathrm{C}_{2}\right)$ ethynylbiphenyl-4- $\left({ }^{15} \mathrm{~N}\right)$ carbonitrile $\left({ }^{15} \mathrm{~N}-{ }^{13} \mathrm{C}_{2}-2\right)$.

Prepared using the procedure described in Ref. 3. The solution of ${ }^{15} \mathrm{~N}^{13} \mathbf{C}_{2} \mathbf{- 4}(0.37 \mathrm{~g}$, $1.34 \mathrm{mmol})$ in methanol $(23 \mathrm{~mL}), 1 \mathrm{~N}$ aqueous potassium hydroxide solution $(1.4 \mathrm{~mL})$ was added and the mixture was stirred at room temperature for $1 \mathrm{~h}$. After removal of methanol, the product was extracted with dichloromethane and recrystallized from dichloromethanehexane to afford ${ }^{15} \mathbf{N}-{ }^{13} \mathbf{C}_{\mathbf{2}} \mathbf{- 2}(275 \mathbf{m g}, 99 \%) .{ }^{1} \mathrm{H}$ NMR $\left(\mathrm{CDCl}_{3}\right) \delta 7.74(\mathrm{~m}, \mathrm{H} 1 / \mathrm{H} 4), 7.68$ (m, H2/H3), 7.60 (m, H6/H7), 7.55 (m, H5/H8), 3.17 (dd, ${ }^{1} \mathrm{~J}_{\mathrm{C} 15-\mathrm{H} 9}=244.1 \mathrm{~Hz},{ }^{2} \mathrm{~J}_{\mathrm{C} 14-\mathrm{H} 9}$ $=57.5 \mathrm{~Hz}, \mathrm{H} 9) .{ }^{13} \mathrm{C} \mathrm{NMR}\left(\mathrm{CDCl}_{3}\right) \delta 144.6(\mathrm{C} 5), 139.4(\mathrm{C} 8), 132.8\left(\mathrm{t},{ }^{2} \mathrm{~J}_{\mathrm{C} 14}={ }^{3} \mathrm{~J}_{\mathrm{C} 15}=2.2\right.$ $\mathrm{Hz}, \mathrm{C} 10 / \mathrm{C} 12), 132.7$ (C3/C7), 127.6 (C4/C6), 127.1 (d, $\left.{ }^{3} \mathrm{~J}_{\mathrm{C} 14}=5.4 \mathrm{~Hz}, \mathrm{C} 9 / \mathrm{C} 13\right), 122.5$ $\left(\mathrm{dd},{ }^{1} \mathrm{~J}_{\mathrm{C} 14}=88.6 \mathrm{~Hz},{ }^{2} \mathrm{~J}_{\mathrm{C} 15}=14.4 \mathrm{~Hz}, \mathrm{C} 11\right), 118.7\left(\mathrm{~d},{ }^{1} \mathrm{~J}_{\mathrm{N} 1}=17.7 \mathrm{~Hz}, \mathrm{C} 1\right), 111.4\left(\mathrm{~d},{ }^{2} \mathrm{~J}_{\mathrm{N} 1}\right.$ $=2.4 \mathrm{~Hz}, \mathrm{C} 2), 83.1\left(\mathrm{~d},{ }^{1} \mathrm{~J}_{\mathrm{C} 15}=176.3 \mathrm{~Hz}, \mathrm{C} 14\right), 78.5\left(\mathrm{~d},{ }^{1} \mathrm{~J}_{\mathrm{C} 14}=176.3 \mathrm{~Hz}, \mathrm{C} 15\right)$.

\section{References}

[1] Pfrommer, B.; Raczkowski, D.; Canning, A.; Louie, S. G. (with contributions from Mauri, F.; Coté, M.; Yoon, Y.; Pickard, C.; Haynes, P.)PARATEC (PARAllel Total Energy Code); Lawrence Berkely National Laboratory: Berkeley, CA. For more information, see: http://www.nersc.gov/projects/paratec.

[2] McNamara, J. M.; Gleason, W. B. J. Org. Chem. 1976, 41, 1071.

[3] Takahashi, S.; Kuroyama, Y.; Sonogashira, K.; Hagihara, N. Synthesis 1980, 627. 